\title{
Elektroaltgeräte als bedeutende Quelle von sekundären Metallen - Entwicklung eines neuen analytischen Verfahrens zur Bestimmung des Metallgehalts
}

\author{
Aleksander Jandric · Stefan Salhofer · Peter Beigl · Marion Huber-Humer
}

Online publiziert: 29. Januar 2018

(C) Der/die Autor(en) 2018. Dieser Artikel ist eine Open-Access-Publikation.

Zusammenfassung Im Vergleich zu Metallerzen der Erdkruste sind Metalle in Elektro- und Elektronikaltgeräten (EAGs) in deutlich höheren Konzentrationen vorhanden, sind aber häufig in komplexe Matrizen unterschiedlicher Metalle oder in Nichtmetall-MetallKomplexe eingebunden. Aufgrund der komplexen Materialzusammensetzung von EAGs, die v.a. durch eine Vielzahl von am Markt verfügbaren Gerätetypen und rasche Technologieentwicklung bedingt ist, wird das volle Recyclingpotenzial derzeit bei weitem noch nicht ausgeschöpft. Davon sind besonders edle und kritische Metalle betroffen, die trotz umfassender Recyclingprozesse mit sehr geringer Effizienz zurückgewonnen werden. Die Erhöhung der Rückgewinnungsrate dieser Metalle erfordert effizientere Methoden und Verfahren. Daraus resultiert der Bedarf an besseren Datengrundlagen zur Charakterisierung von EAGs.

Im Rahmen dieser Forschungsarbeit wurden besonders wertvolle Bauteile aus EAGs durch eine Tiefenzerlegung und anschließend mit einer Reihe von einfachen Methoden analysiert, wie z.B. Nickeltest, Aluminium/ Magnesium-Test, um einen möglichst praktikablen Ansatz für die Materialanalyse von EAGs bereitzustellen.

Die vorgestellten Ergebnisse basieren einerseits auf Literaturrecherchen, anderseits auf den Ergebnissen der durchgeführten Versuche zur Bestimmung der Materialzusammensetzung von EAGs. Dadurch ist es möglich, die besonders wertvollen Typen von

DI A. Jandric $(\bowtie)$.

ao. Univ.-Prof. DI Dr. S. Salhofer .

Mag. DI P. Beigl •

Univ.-Prof. DI Dr. M. Huber-Humer Institut für Abfallwirtschaft,

Universität für Bodenkultur Wien,

Muthgasse 107/III, 1190 Wien,

Österreich

aleksander.jandric@boku.ac.at
EAGs bzw. deren Bauteile in Bezug auf edle und kritische Metalle zu bestimmen. Anschließend werden neben dem Materialgehaltpotenzial auch die Herausforderungen und Potenziale für die Bestimmung der Materialzusammensetzung von EAGs diskutiert.

Waste electrical and electronic equipment as a significant source of secondary metals -

Development of a new analytical method for the determination of metal content

Abstract In contrast to metal ores, metals from Waste Electrical and Electronic Equipment (WEEE) are present in significantly higher concentrations, but are often bounded in complex matrices of different metals or in non-metal/ metal complexes. Due to the complex material composition of WEEE, i. e. due to a myriad of device types available on the market and rapid technology development, full recycling potential is far from being achieved. As a result, special precious and critical metals are affected, which despite extensive recycling processes are currently recovered with very low efficiency. Increasing the recovery rate of these metals requires more efficient methods and procedures. This results in the need for better data bases for the characterization of WEEE.

In the context of this research, particularly valuable components from WEEE were analysed by in-depth dismantling and subsequently identified by a number of simple analytical tests, e.g. nickel test, aluminium/magnesium test, in order to present an applicable approach for material analysis of WEEE.

The results are based on literature research on the one hand, and on the results of the experiments carried out to determine the composition of WEEE on the other hand. This approach allows to determine the most valuable waste electrical and electronic devices or their components in relation to noble and critical metals. Next to the material content potential, the challenges and potentials for the determination of the material composition of WEEE will be discussed.

\section{Einleitung}

Das weltweite Aufkommen an Elektroaltgeräten (EAGs) betrug im Jahr 2014 mehr als 48Mio. Tonnen. Mit einer jährlichen Wachstumsrate von 4 bis $5 \%$ sind EAGs der am schnellsten wachsende Abfallstrom und schon im Jahr 2018 wird diese Menge auf 50Mio. t steigen (Baldé et al. 2015). Nachdem die Weltbevölkerung im Jahr 2014 ungefähr 7,266 Mrd. Menschen umfasste (Worldometers 2017), betrug das globale durchschnittliche EAG-Aufkommen 5,5 kg pro Kopf und Jahr. Das EAG-Aufkommen wird von mehreren in Wechselbeziehung zueinanderstehenden Faktoren beeinflusst. Zum einen besteht eine direkte Korrelation zwischen dem Bruttoinlandsprodukt pro Kopf (BIP) und der steigenden Nachfrage nach Gütern und Dienstleistungen, was in der Folge zu einem höheren Abfallaufkommen führt (Huisman et al. 2012). Zum anderen hat die intensive technologische Weiterentwicklung in Verbindung mit einer schnellen globalen Marktdurchdringung dazu geführt, dass elektronische Geräte nach sehr kurzer Zeit entsorgt werden. Ein weiterer, weniger signifikanter Faktor ist das Bevölkerungswachstum. Die Nutzung von Technologie und Zugang zum Internet ist zu einer Notwendigkeit für alltägliche Funktionen in der modernen Gesellschaft geworden und verstärkt somit die steigende Nachfrage nach elektronischen Geräten.

Elektrische und elektronische Geräte enthalten zahlreiche Materialien, die aufgrund deren Auswirkungen auf die Umwelt als Schadstoffe definiert sind. Beispielsweise enthält die Unterkate- 
gorie der Elektrokleingeräte folgende durchschnittliche Konzentrationen an Schwermetallen [in $\mathrm{mg} / \mathrm{kg}$ ]: $210 \mathrm{Cd}$, $130 \mathrm{Co}, 800 \mathrm{Ni}, 1780 \mathrm{~Pb}, 310 \mathrm{Sn}$ (Fu et al. 2008). Neben Schwermetallen enthalten Elektrokleingeräte eine Vielzahl weiterer gefährlicher Bestandteile wie z.B. Flammschutzmittel (TBBPA, HBCD, TetraBDE usw.), organische Schadstoffe (BTEX, PAK, PCB etc.) und Flüssigkristalle (Salhofer und Tesar 2011). Ein Großteil sind flüchtige und reaktive Stoffe und ihre Entsorgung auf Deponien ohne vorherige Behandlung kann zu Umwelt- und Gesundheitsproblemen führen. Solche Materialien, die von Natur aus ein Gefährdungspotenzial darstellen und direkt aus EAGs stammen, werden als "primäre Schadstoffe“ definiert (vgl. Khanna et al. 2014). Darüber hinaus treten Emissionen bei der Behandlung von EAGs als Folge von sowohl formellen als auch informellen Recyclingtechniken auf. Diese Emissionen können als „sekundäre Schadstoffe" klassifiziert werden. Bei der Zerkleinerung von EAGs wird unter anderem Staub freigesetzt, der Cadmium, Blei, PBDE, TBBPA und andere Stoffe enthält. Die (offene) Verbrennung und pyrometallurgische Verarbeitung führen $\mathrm{zu}$ heterogenen Emissionen, die zum Teil aus flüchtigen Substanzen bestehen.

Die Behandlung von EAGs wurde zunächst hauptsächlich als Umweltproblem angesehen. In den letzten Jahren werden EAGs zunehmend auch unter dem Gesichtspunkt der Geopolitik und der Verteilung natürlicher Ressourcen betrachtet. Da die Bauteile von Elektro- und Elektronikgeräten aus einer Vielzahl an Metallen bestehen, wird zunehmend das Ressourcenpotenzial wahrgenommen. Einzelne Geräte, wie z.B. Mobiltelefone, können mehr als 20 verschiedene Metalle enthalten, von denen viele zur Kategorie der Edelmetalle, Platinmetalle oder der Metalle der „Seltenen Erden“ gehören (Schluep et al. 2009). Die höchsten Konzentrationen von Edelmetallen und Seltenen Erden sind in Leiterplatten enthalten, vor allem in PC-Motherboards und Leiterplatten aus Mobiltelefonen, integrierten Schaltkreisen, Festplattenlaufwerken, Schaltern und Verbindungssteckern.

Obwohl der Edelmetallgehalt in einem einzigen Gerät nur in der Größenordnung von bis $\mathrm{zu}$ einigen hundert Milligramm liegt, ergeben sich aufgrund der Gesamtmasse der EAG insge- samt signifikante Reserven an wertvollen Materialien in diesem Abfallstrom.

Ziel dieser Forschungsarbeit ist die Entwicklung eines analytischen Verfahrens zur Bestimmung der Materialzusammensetzung von EAGs. Um dieses Ziel zu erreichen, wurde ein innovatives Materialidentifikationsverfahren basierend auf einfachen Tests zur Metallerkennung erstellt, um die Konzentrationen von Spurenelementen zu bestimmen. Zudem wird auch der Einfluss der technologischen Entwicklung auf die Änderung der Materialzusammensetzung von analysierten Bauteilen näher untersucht.

\section{Rechtliche Rahmenbedingungen}

Der rechtliche Rahmen für die Entsorgung von EAGs in der EU beruht im Wesentlichen auf zwei Richtlinien: Richtlinie 2012/19/EU für Elektroaltgeräte, sog. WEEE-Richtlinie (Waste of Electrical and Electronic Equipment), und Richtlinie 2011/65/EU (RoHS) zur Beschränkung der Verwendung bestimmter gefährlicher Stoffe in Elektro- und Elektronikgeräten, sog. RoHS-Richtlinie (Restriction of Hazardous Substances). Die Richtlinien traten 2003 bzw. 2006 in Kraft und haben seither den EAGRecyclingmarkt in der EU, im Europäischen Wirtschaftsraum und darüber hinaus entscheidend geprägt. Die WEEE-Richtlinie regelt die Sammlung und Behandlung von EAGs und wurde seit ihrem Inkrafttreten im Jahr 2003 mehrmals novelliert. Die letzte Novellierung der WEEE-Richtlinie trat im Jahr 2012 in Kraft. Kernelemente der WEEERichtlinie umfassen die Einteilung der EAGs in relevante Unterkategorien zur Festlegung der Sammel- und Recyclingquoten sowie die Exporte von EAGs (vgl. Salhofer et al. 2016; Huisman et al. 2007).

Die Novellierung der WEEE-Richtlinie von 2012 sieht vor, ab August 2018 die derzeit relevanten zehn WEEE-Kategorien in sechs neue Unterkategorien zu überführen. Die neuen Kategorien sind: 1) Wärmeüberträger, 2) Bildschirme und Monitore, 3) Lampen; 4) Großgeräte, 5) Kleingeräte und 6) Kleine ITund Telekommunikationsgeräte (keine äußere Abmessung beträgt mehr als $50 \mathrm{~cm}$ ). Darüber hinaus wird die Sammelquote von Elektro- und ElektronikAltgeräten auf $45 \%$ der gesamten in Verkehr gebrachten Elektro- und Elektronikgeräte festgelegt. Die Sammelquote wird berechnet als Verhältnis des Gesamtgewichts der gesammelten EAGs zum durchschnittlichen Gewicht der Elektrogeräte, die in den drei vorangegangenen Jahren auf den Markt gebracht wurden. Recyclingziele werden für jede Unterkategorie einzeln festgelegt und liegen zwischen 55 und $85 \%$.

Trotz der in der WEEE-Richtlinie genannten Maßnahmen zeigt die Praxis, dass immer noch große Mengen an EAGs global betrachtet ohne adäquate Behandlung entsorgt werden (Tran und Salhofer 2016; Baldé et al. 2015). Insofern bergen Schwermetalle und Flammschutzmittel in den EAGs ein erhebliches Gesundheits- und Umweltrisiko. Dieser Problematik sollte mit dem Inkrafttreten der RoHS-Richtlinie entgegnet werden, welche den Einsatz bestimmter gefährlicher Materialien bei der Herstellung von Elektro- und Elektronikgeräten reguliert. Von der RoHSRichtlinie geregelte Materialien umfassen Blei, Quecksilber, Cadmium, sechswertiges Chrom, polybromierte Biphenyle, polybromierte Diphenylether und Phthalate.

Neben den angeführten Richtlinien stellt die Rohstoffinitiative „Sicherung der Versorgung Europas mit den für Wachstum und Beschäftigung notwendigen Gütern" eine weitere wichtige Grundlage für die Ausrichtung der Recyclingbranche in der EU dar. Gegründet wurde diese Initiative von der Adhoc-Working-Group (Untergruppe) der Rohstoffversorgungsgruppe, und ihre Hauptaufgabe liegt darin, die Kritikalität von Rohstoffen in der EU zu beurteilen (Europäische Kommission 2017). Die geopolitischen und wirtschaftlichen Veränderungen haben dabei einen wesentlichen Einfluss auf die Verfügbarkeit bestimmter Rohstoffe. Die Rohstoffe werden als kritisch beurteilt, wenn das Risiko für Versorgungsengpässe und deren Auswirkungen auf die Wirtschaft größer sind als bei den meisten anderen (Luidold et al. 2013). Die erste Liste der kritischen Rohstoffe (engl. Critical Raw Materials) wurde im Jahr 2011 erstellt und beinhaltete vierzehn Rohstoffe. Mittlerweile wurde die Liste zwei Mal aktualisiert, das letzte Mal im Jahr 2017, und wurde auf 27 kritische Rohstoffe erweitert, die in Abb. 1 als rote Punkte hervorgehoben sind. 


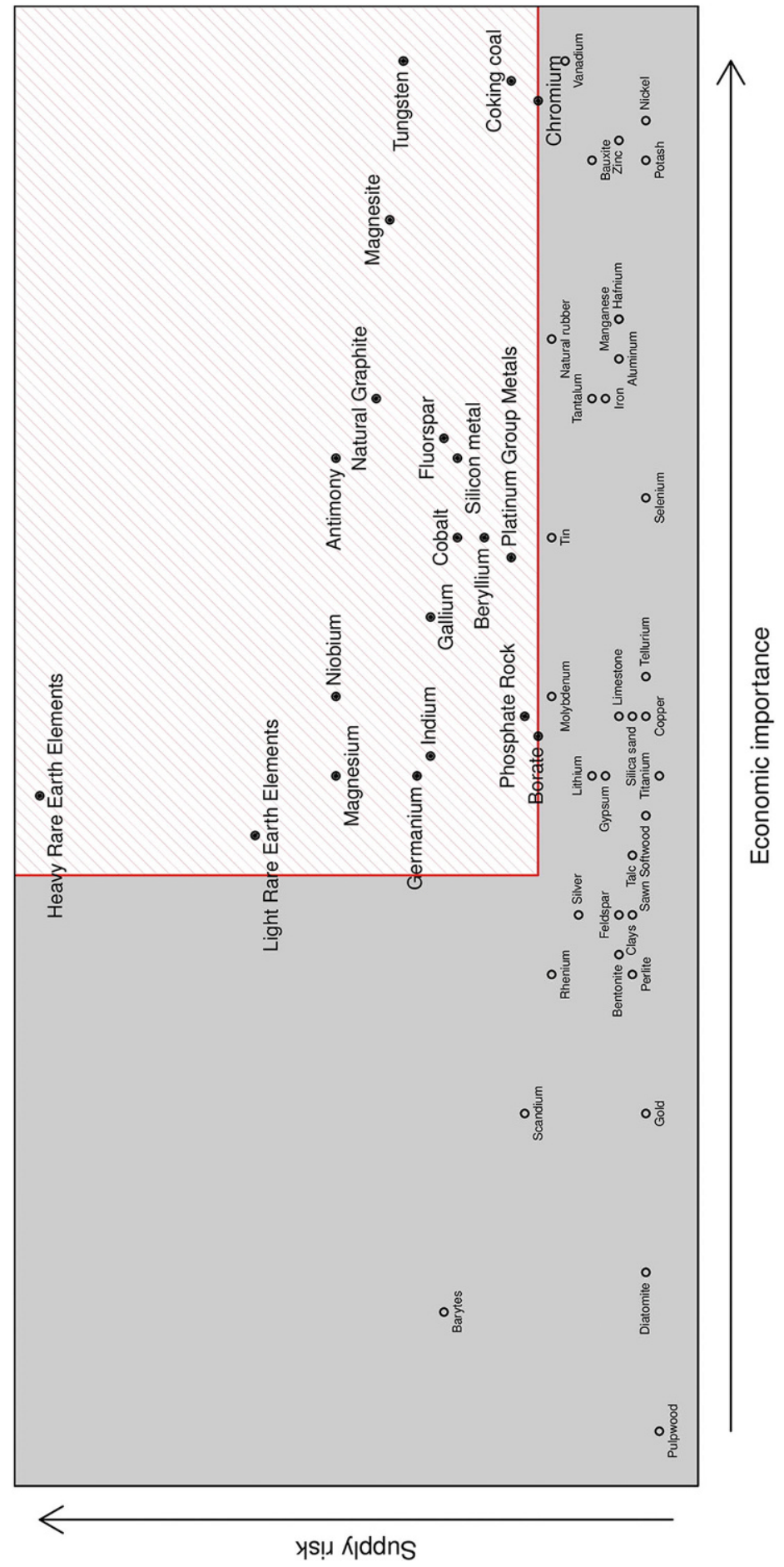

\section{Material und Methoden zur Untersuchung komplexer Bauteile}

Fokus der vorgestellten Forschungsarbeit waren die Bauteile, die ein erhebliches Rohstoffpotenzial aufweisen, aber aufgrund ihrer komplexen Zusammensetzung aus Sicht der Verwertungsprozesse nicht mit Monomaterialien wie Eisenmetalle oder Aluminium verglichen werden können. Aus praktischen Gründen werden diese Bauteile in Folge vereinfachend als „komplexe Bauteile" bezeichnet. Der Materialstrom, der diese komplexen Bauteile enthält, resultiert hauptsächlich aus dem Zerlegungsprozess und der mechanischen Schadstoffentfrachtung. Obwohl komplexe Bauteile auch in den nachfolgenden Prozessschritten anfallen (beispielsweise als Outputmaterial aus dem Shredder), werden Bauteile in diesen Prozessen in der Regel zerkleinert und verformt, was eine anschließende Analyse erheblich erschwert.

Die Wirtschaftlichkeit der Vorbehandlung und die Recyclingeffizienz der Verwertung stehen in direktem $\mathrm{Zu}$ sammenhang mit der Fähigkeit des Recyclingverfahrens, die Materialströme möglichst homogen zu trennen. Als allgemeine Regel für die Vorbehandlung gilt, dass die Rückgewinnungsrate eines bestimmten Metalls mit der zunehmenden Inhomogenität dieses Metalls im Ausgangsmaterialstrom abnimmt. Das bedeutet: Je komplexer die Materialzusammensetzung, desto geringer ist die Recycling-Effizienz (Chancerel et al. 2009; Hagelüken und Corti 2010). Schließlich bestimmt die Homogenität des Outputstroms aus der Vorbehandlung, in Kombination mit den aktuellen Marktpreisen, den Verkaufspreis dieses Outputstroms.

Im folgenden Kapitel wird die methodische Vorgehensweise der Analyse komplexer Bauteile aus EAGs beschrieben, die im Rahmen einer Masterarbeit am Institut für Abfallwirtschaft der Universität für Bodenkultur Wien entwickelt und durchgeführt wurde (Jandric 2017). Das angewendete analytische Verfahren nützt die komplementäre Anwendung einer einfachen Materialidentifikationsanalyse für über 80 unterschiedliche Bauteile aus EAGs sowie ein innovatives Verfahren mittels ICP-OES-Analyse für die Bestimmung des Spurenelementgehalts in z.B. Kondensatoren. Die Ergebnisse zur ICPOES-Analytik sind in Jandric (2017) zu 


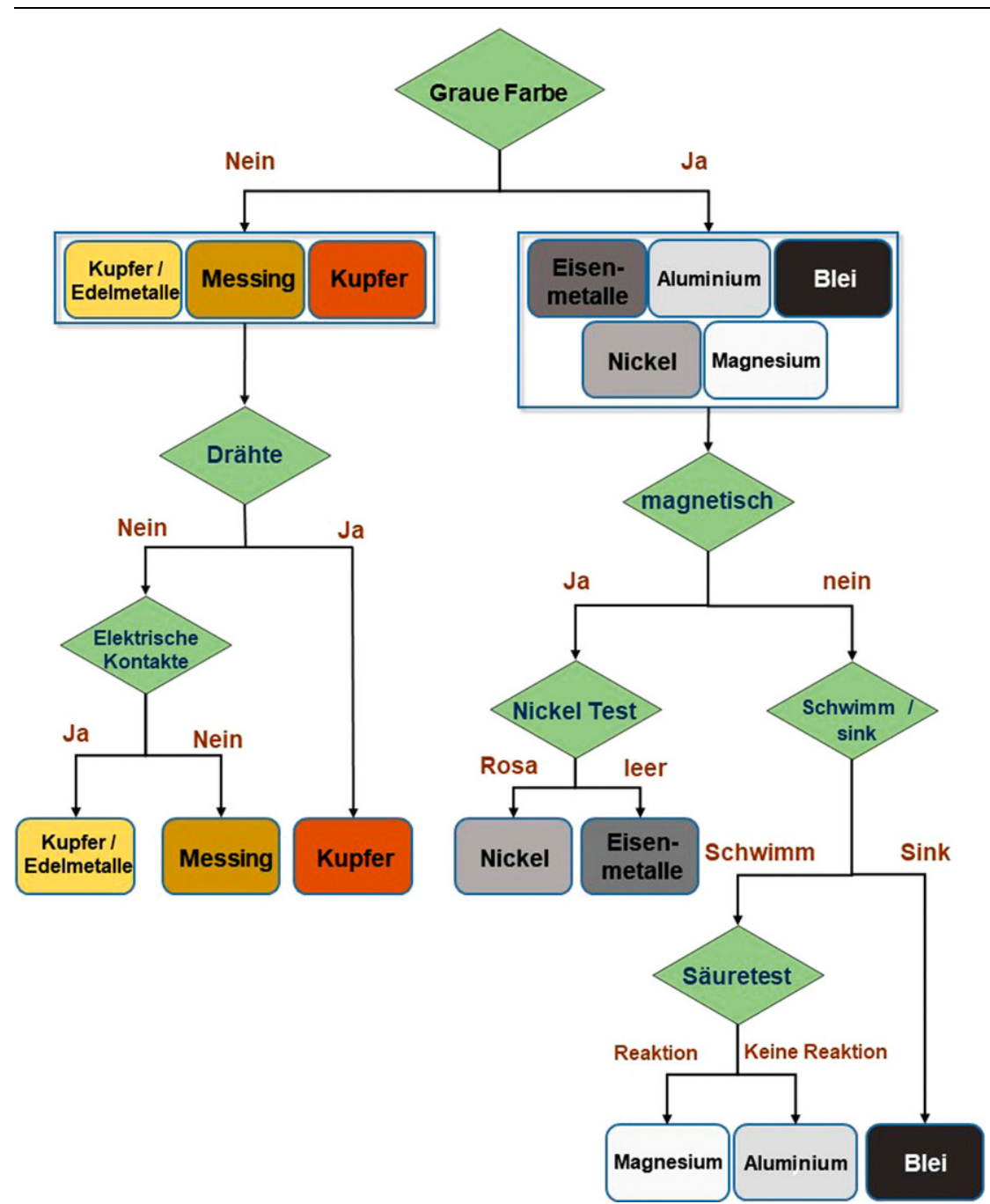

Abb. 2 Metallbestimmung im Rahmen der manuellen Tiefenzerlegung

finden und werden in diesem Beitrag nicht explizit dargestellt.

Die Zielbauteile für die Analyse wurden aufgrund der Verfügbarkeit ihrer Materialzusammensetzungen in der wissenschaftlichen Literatur und ihrer wirtschaftlichen Bedeutung ausgewählt. Grundlage für die Analyse waren die folgenden Bauteile: Festplattenlaufwerke, Netzteile aus PCs, Leiterplatten, optische Laufwerke, Netzteile für Drucker, aktive Lüfter und Aluminiumkondensatoren. In der vorliegenden Arbeit werden die Ergebnisse für die ersten drei Bauteiletypen vorgestellt (Festplattenlaufwerke, Netzteile aus PCs und Leiterplatten).

In einem ersten Schritt wurde eine manuelle Tiefenzerlegung der Bauteile vorgenommen. Das Ziel der Analyse bestand darin, die Zielbauteile in einzelne Materialien $\mathrm{zu}$ zerlegen und so $\mathrm{zu}$ trennen, dass sie identifiziert und ho- möglich, werden die Teile der ersten Ebene (Funktionsebene) in einzelne homogene Materialgruppen zerlegt. Mit diesem Verfahren ist es möglich, die potenziellen Änderungen in der Materialzusammensetzung komplexer Bauteile zwischen Gerätetypen genau $\mathrm{zu}$ lokalisieren wie auch die Veränderung der Materialzusammensetzung über die Jahre zu verfolgen.

Der Magnettest wird verwendet, um magnetische von nichtmagnetischen grauen Metallen zu unterscheiden. Die magnetischen Fraktionen umfassen Eisenmetalle, Nickel und Ferrit. Es ist ziemlich einfach und wichtig, Ferrit von den anderen Metallen zu trennen, da Ferrit eine keramische Verbindung aus Eisenoxid $\left(\mathrm{Fe}_{2} \mathrm{O}_{3}\right)$ ist. Ferrite werden gewöhnlich als Kern in Transformatoren oder als Permanentmagnete verwendet.

Der Nickel-Test wird verwendet, um Eisen- und Nickelfraktionen zu unterscheiden. Da beide Metalle magnetisch sind und eine ähnliche Dichte haben, müssen zusätzliche Tests durchgeführt werden, um sie voneinander unterscheiden zu können. Zu diesem Zweck wird ein Tropfen 10\%iger Ammoniak, gefolgt von einem Tropfen Dimethylglyoxim, auf ein Wattestäbchen getropft, welches für 15 bis $20 \mathrm{~s}$ gegen die Oberfläche des Metalls gerieben wird. Verfärbt sich das Wattestäbchen rosa oder rot, ist dies ein Zeichen für das Metall Nickel. Wenn sich die Farbe des Wattestäbchens nicht ändert, handelt es sich um eine Eisenmetallfraktion.

Der Schwimm-/Sink-Test wird durchgeführt, um die nichtmagnetischen Metalle zu unterscheiden. $\mathrm{Zu}$ diesem Zweck wurde eine Natriumpolywolframatlösung mit einer Dichte von $3,1 \mathrm{~g} / \mathrm{cm}^{3}$ verwendet. Aluminiumund Magnesiummetalle, deren Dichte $2,8 \mathrm{~g} / \mathrm{cm}^{3}$ nicht übersteigt, schwimmen auf der Oberfläche der Lösung auf. Das Blei, das eine Dichte von $10,6 \mathrm{~g} / \mathrm{cm}^{3}$ hat, sinkt jedoch schnell in der Lösung ab. Dieser Test zeigt zufriedenstellende Ergebnisse, solange die Stücke nicht zu klein sind. In dem Fall kann es vorkommen, dass die Oberflächenspannung der Lösung verhindert, dass ein dichteres Metall absinkt.

Der Säuretest wird durchgeführt, um Magnesium von Aluminium zu unterscheiden. $\mathrm{Zu}$ diesem Zweck wurde ein sehr einfacher Test mit Apfelessig durchgeführt. Es wurden wenige Tropfen Essig direkt auf die Oberfläche des Metalls getropft. Das Magnesium reagiert unmittelbar im Kontakt mit dem 


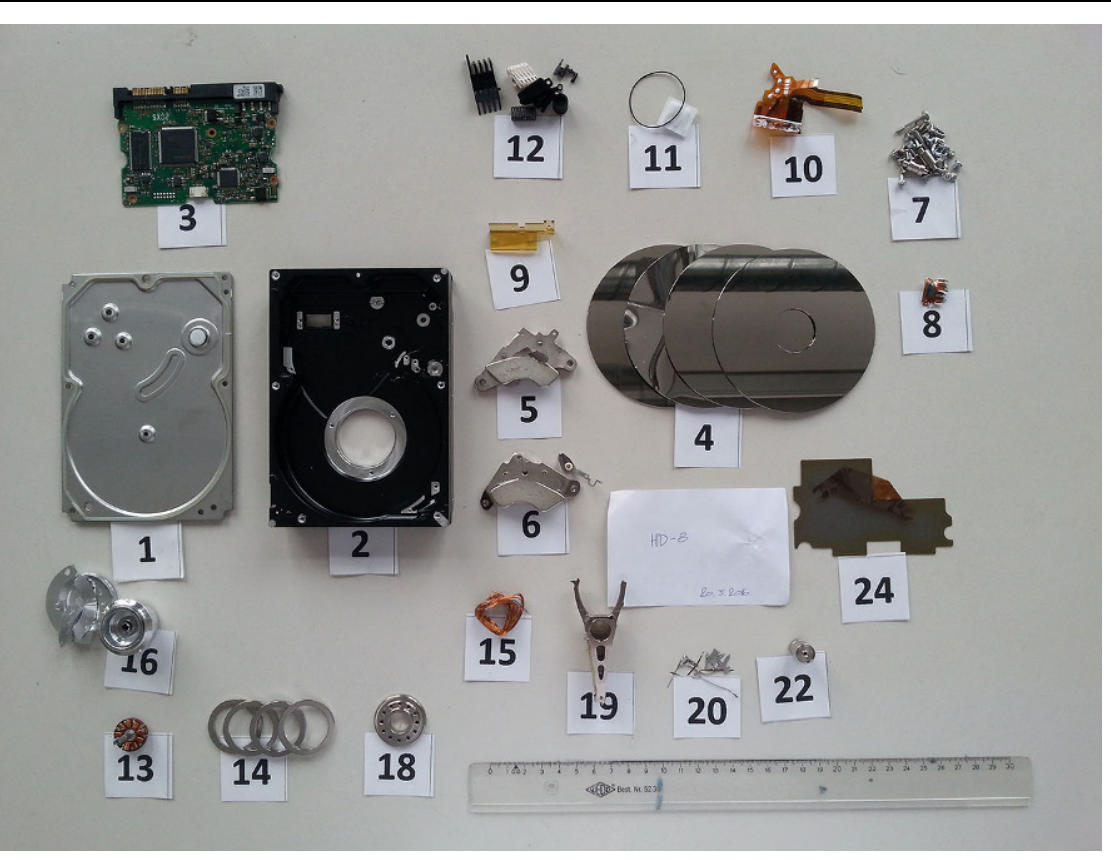

Abb. 3 Beispiel eines demontierten Festplattenlaufwerks

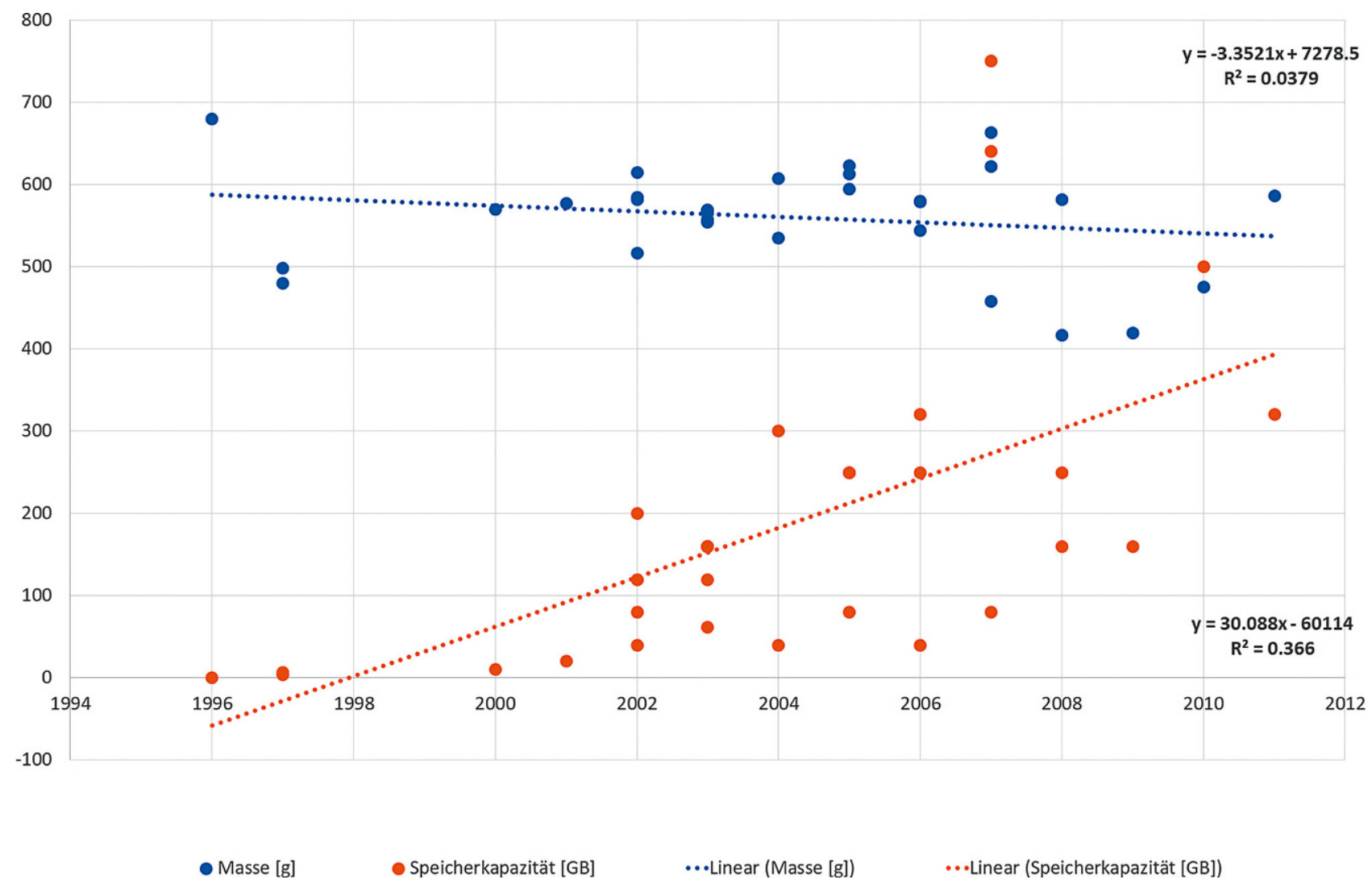

Abb. 4 Die Gesamtmasse und Speicherkapazität der analysierten Festplattenlaufwerke ( $n=32)$; y-Achse: g bzw. GB; x-Achse: Jahreszahl 


\begin{tabular}{|c|c|c|c|c|c|c|c|c|c|c|c|c|c|}
\hline \multicolumn{11}{|c|}{$\begin{array}{l}\text { Basismetalle und kritische Metalle } \\
{[\%]}\end{array}$} & \multicolumn{3}{|c|}{$\begin{array}{l}\text { Edelmetalle } \\
{[\mathrm{ppm}]}\end{array}$} \\
\hline Al & $\mathrm{Cu}$ & $\mathrm{Fe}$ & $\mathrm{Ni}$ & Sn & $\mathrm{Ti}$ & $\mathrm{Zn}$ & $\mathrm{Ce}$ & $\mathrm{Nd}$ & $\mathrm{Sb}$ & $\mathrm{Ta}$ & $\mathrm{Ag}$ & $\mathrm{Au}$ & $\mathrm{Pd}$ \\
\hline 2,2 & 31,6 & 7,1 & 2,5 & 2,4 & 0,6 & 0,4 & 0,5 & 0,2 & 0,1 & 0,8 & 3,340 & 1,020 & 210 \\
\hline
\end{tabular}
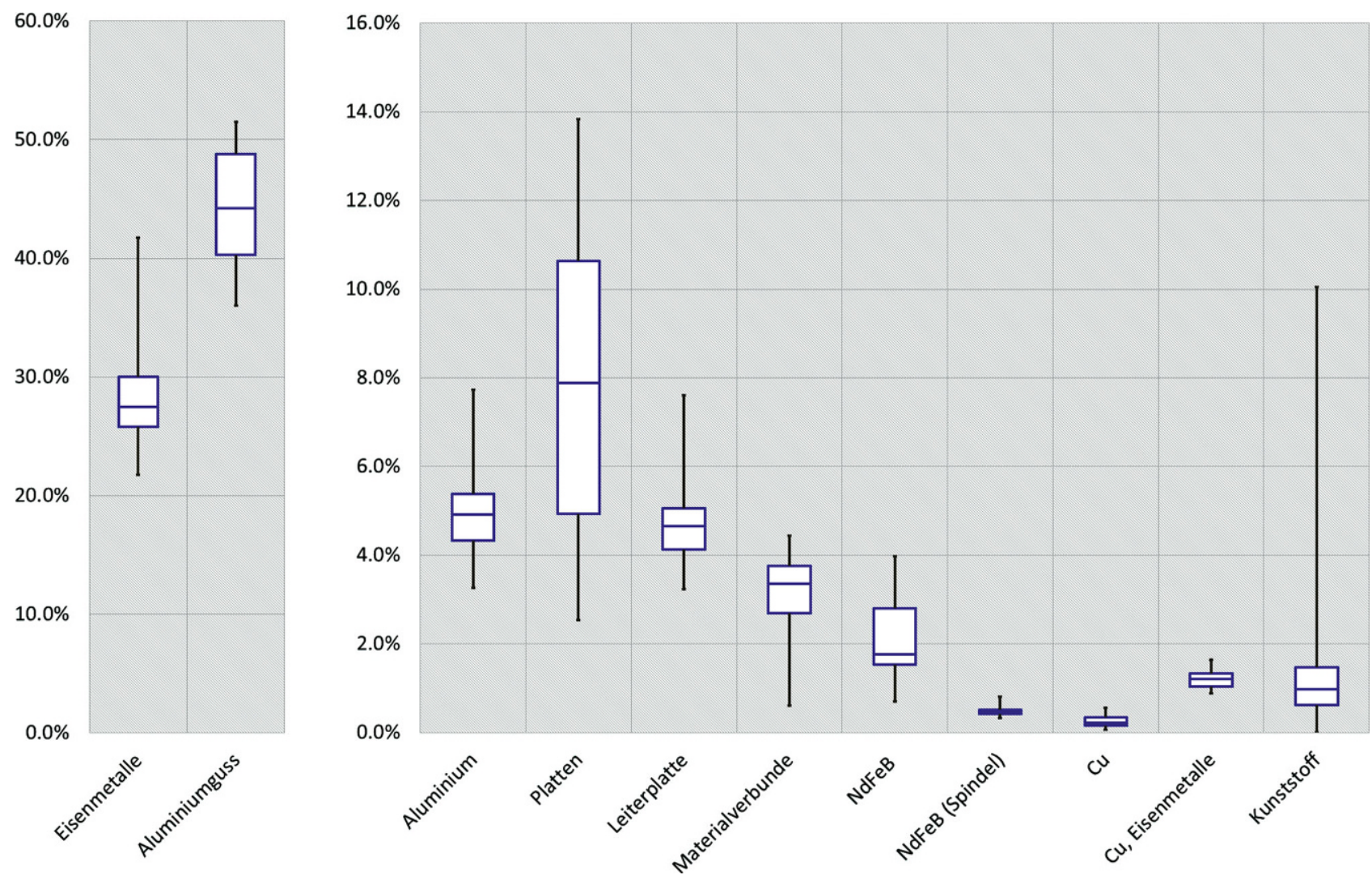

Abb. 5 Masse und Materialzusammensetzung von Bauteilen aus Festplattenlaufwerken $(n=32)$

Essig. Im Gegensatz dazu zeigt Aluminium keine sichtbaren Anzeichen einer Reaktion.

Darüber hinaus ermöglicht dieses vereinfachte Vorgehen die Ergänzung der Ergebnisse mit Daten aus der Literatur für Bauteile, deren genaue Materialidentifikation den Umfang der Analyse übersteigen würde, was für Leiterplatten, Magnete, Platten von Festplattenlaufwerken und ähnliche Bauteile meistens der Fall ist.

\section{Materialzusammensetzung von ausgewählten EAG-Bauteilen}

\subsection{Festplattenlaufwerke}

Im Rahmen der vorliegenden Arbeit wurden PC Festplattenlaufwerke analysiert, die zwischen den Jahren 1996 und 2011 hergestellt wurden. Die durchschnittliche Masse pro Festplattenlaufwerk beträgt $559 \pm 61 \mathrm{~g}$. Obwohl die Speicherkapazität von Festplattenlaufwerken seit Mitte der 1990erJahre deutlich zugenommen hat, hat sich das durchschnittliche Gewicht von Festplattenlaufwerken aufgrund von Effizienzsteigerungen nicht wesentlich verändert.

Das Gehäuse eines Festplattenlaufwerks besteht aus zwei Teilen: Basisteil und Deckel (siehe Abb. 3). In allen analysierten Proben bestand das Basisteil aus Aluminiumguss mit einem durchschnittlichen Gewicht von $246 \pm 23 \mathrm{~g}$. Bei 10 von 32 der analysierten Festplattenlaufwerke bestand auch die Abdeckung aus Aluminium, die anderen 22 der 32 Festplattenlaufwerke hatten eine Abdeckung aus Eisenmetall. Die Hülle hat eine Masse von $113 \pm 28$ g. Das Gehäuse macht mehr als 70 Gew.-\% der gesamten Festplattenlaufwerke aus und hat somit einen signifikanten Einfluss auf das Gesamtgewicht des Festplattenlaufwerks. Es konnte weder ein zuneh- mender noch ein abnehmender Trend für die Masse von Festplatten-Gehäusen über die Zeit festgestellt werden (Abb. 4).

Teile, die unter die Unterkategorie Plattenstapelanordnung fallen, umfassen (Speicher-)Platten, einen Abstandhalter zwischen den Platten und eine Spindelabdeckung. Die Platten variieren in Größe und Anzahl von einem bis zu vier Plattenteller pro Festplattenlaufwerk. Die Platten haben einen Aluminiumkern mit einer sehr dünnen Schicht einer Metalllegierung, die Cr, $\mathrm{Co}, \mathrm{Ni}, \mathrm{Fe}$ und $\mathrm{Zn}$ enthält, um magnetische Eigenschaften herzustellen, die für die Datenspeicherung notwendig sind (Habib et al. 2015). Bei Betrachtung aller analysierten Festplattenlaufwerke kann eine Zunahme im Gewicht der Plattenstapelanordnung beobachtet werden, was durch die zunehmende Speicherkapazität und durch den Be- 


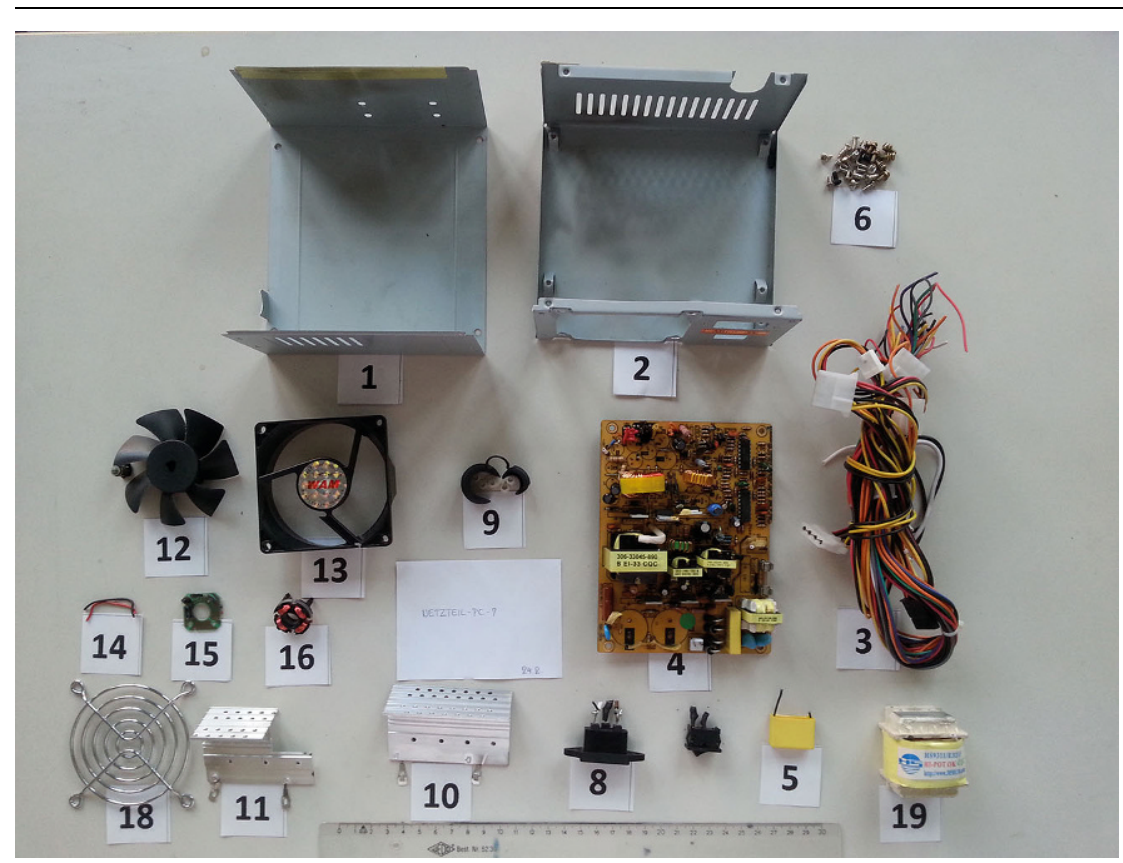

Abb. 6 Beispiel eines demontierten Netzteils

darf für mehr Platten erklärt werden kann.

Festplattenlaufwerke haben nur eine Leiterplatte, die an der Außenseite des Gehäuses befestigt ist. Eine Leiterplatte wiegt im Durchschnitt $26,2 \pm 5 \mathrm{~g}$. Es kann ein klarer Trend in der Größe und Gewichtsreduktion von Leiterplatten bestimmt werden. Das mittlere Gewicht für Leiterplatten, hergestellt zwischen 1996 und $2002(n=9)$, beträgt 29,9g, während das mittlere Gewicht von Leiterplatten, hergestellt zwischen 2007 und 2011 ( $n=9), 22,4 \mathrm{~g}$ beträgt. Ein detaillierter Überblick über die Materialzusammensetzung von Leiterplatten aus Festplatten ist in Tab. 1 gegeben. Die Studie von Ueberschaar und Rotter (2015) wurde ausgewählt, weil die Studie im Gegensatz zum Großteil der bestehenden Literatur detaillierte Informationen über die Probengröße, Analyseverfahren und Abweichungen in der Materialzusammensetzung enthält.

Neben Leiterplatten wurde in dem Bauteil Schwingspule (engl. Voice-CoilAssembly) und Mechanik ein deutlicher Trend bei der Gewichtsabnahme beobachtet. Die Schwingspule besteht aus Neodym-Permanentmagneten und Eisenmetallhaltern, während die Unterkategorie Mechanik Schrauben, Kunststoffstoßfänger, Aufkleber und Kunststoffpolsterisolatoren umfasst.

Festplattenlaufwerke bestehen hauptsächlich aus Metallteilen (über 90 Gew.$\%$ ), die restlichen $10 \%$ sind metallische

\subsection{Netzteile aus PCs}

Es wurden 10 verschiedene Netzteile von Desktop-Computern (PCs) analysiert. Die analysierten Netzteile haben ein durchschnittliches Gewicht von $1460 \pm 352$ g. Es konnte kein eindeutiger Trend in der Änderung der Gesamtmasse festgestellt werden.

Bauteile, die ein Netzteil bilden, sind Gehäuse, Leiterplatte, Kabel, passiver Kühlkörper (Aluminium), aktiver Lüfter (Kunststoff) und eine Reihe von Bauteilen, die von Modell zu Modell erheblich variieren können (wie Transformatoren, Induktivitäten, Schalter usw.). In 4 von 10 Netzteilen wurde der Haupttransformator auf der Leiterplatte montiert, in den anderen 6 der 10 untersuchten Fälle wurde er als separater Bauteil in das Netzteil eingebaut. Wenn der Transformator als separater Bauteil vorhanden war, wurde auch das Gewicht getrennt erhoben. In allen anderen Fällen wurde es zusammen mit der Leiterplatte gewogen, auf der es montiert ist (siehe Abb. 6).

Die Bauteile auf der Netzteil-Leiterplatte sind im Vergleich zu anderen Leiterplatten sehr groß. Sie umfassen verschiedene Kondensatoren, von denen Radial- und Aluminiumkondensatoren die größten sind, verschiedene Arten von elektromagnetischen Spulen (z.B. Transformatoren, Induktoren usw.), Dioden, Sicherungen und nur wenige integrierte Schaltungen. Darüber hinaus hat die Platine des Netzteils passive Kühlkörper aus Aluminium, die separat ausgebaut und gewichtet wurden. Alle anderen Bauteile stellen Materialkomplexe (engl. Assemblies) dar und wurden nicht einzeln verwogen (siehe Abb. 7).

In Bezug auf die Materialzusammensetzung ist der größte Materialanteil von Netzteilen eine Eisenmetallfraktion, die das Gehäuse von PSU bildet. Fast alle anderen Materialfraktionen werden als Metall-Nicht-Metall-Verbunde wie PCB, Kabel („Cu, Kunststoff“ in Abb. 7) und andere dargestellt. Die Unterkategorie Materialverbunde („Assemblies“) in Abb. 7 setzt sich auch aus Materialverbunden aus Metall und Nichtmetall zusammen und umfasst Transformatoren, Stecker, Schalter und andere Bauteile, die nicht auf der Leiterplatte montiert sind. Wie erwartet, zeigt diese Fraktion eine bemerkenswert hohe Streurate aufgrund der Unregelmäßigkeit in Größe und Art der konstituierenden Bauteile. 


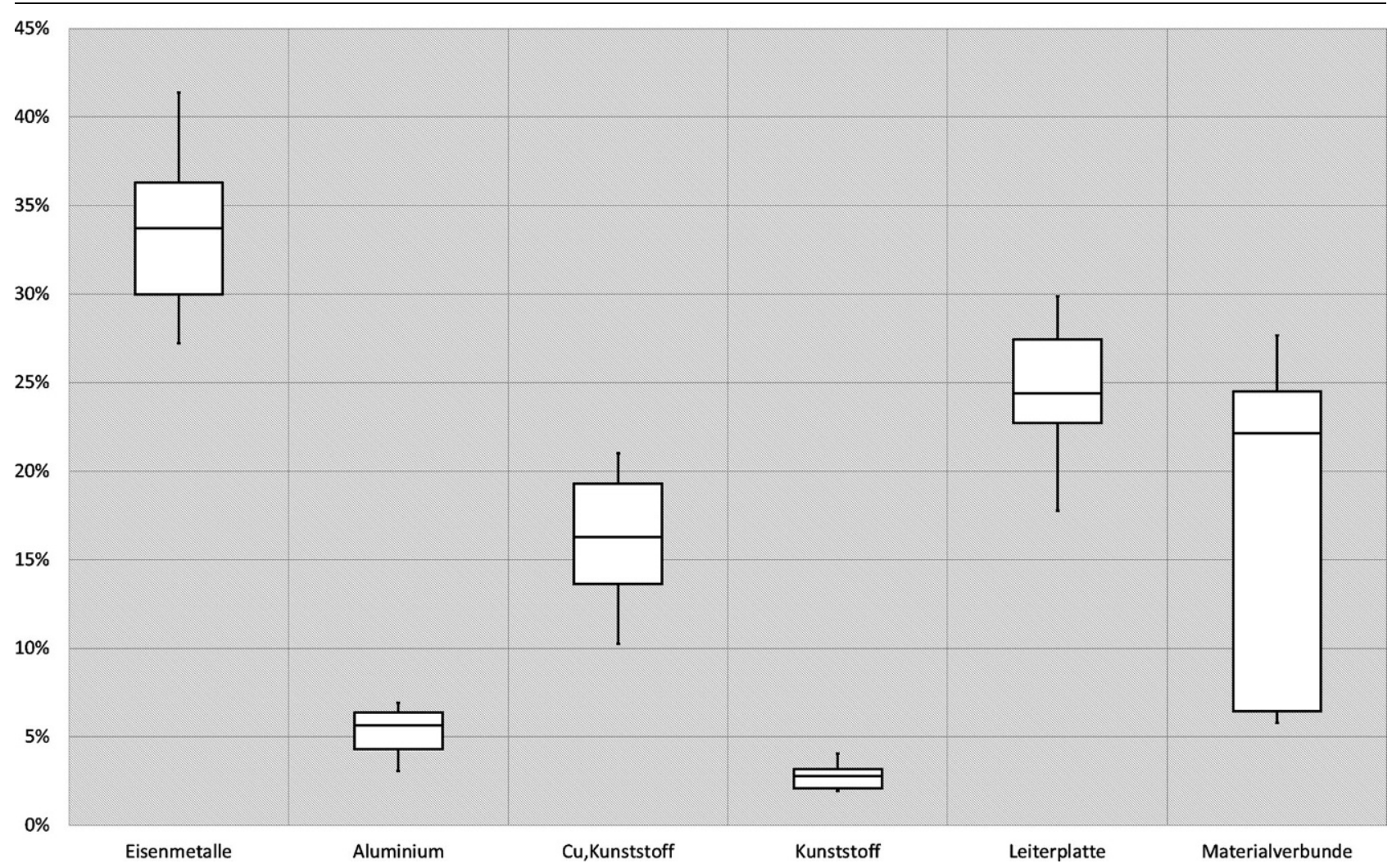

Abb. 7 Materialzusammensetzung von Netzteilen $(n=10)$

\subsection{Leiterplatten}

Leiterplatten sind komplexe Materialverbindungen und haben aufgrund ihres Gehalts an Kupfer und Edelmetallen ein hohes Recyclingpotenzial. Leiterplatten enthalten allerdings auch Schwermetalle, wie z. B. Pb, Zn, Sb und andere Materialien, die zu negativen Umweltauswirkungen führen. Aus diesem Grund werden Leiterplatten gemäß der europäischen WEEE-Richtlinie als "gefährliche Abfälle“ eingestuft.

Basierend auf den Konstruktionsverfahren werden Leiterplatten in drei Hauptgruppen eingeteilt: einseitig, doppelseitig und mehrschichtig. Die einseitigen Platinen haben nur auf einer Seite der Leiterplatte eine leitfähige (Kupfer-)Schicht, während die doppelseitigen auf beiden Seiten der Leiterplatte leitende Schichten aufweisen. Die mehrschichtigen Leiterplatten weisen zwei oder mehr leitende Schichten auf, die durch das nichtleitende Material getrennt sind. Mehr leitfähige Schichten ermöglichen eine dichtere Besetzung der Leiterplatte mit integrierten Schaltungen (IC), Kondensatoren, Dioden usw.
Abb. 8 zeigt einen Überblick über die $\mathrm{Cu}$ - und $\mathrm{Au}$-Konzentration in Leiterplatten. Das Review umfasst eine Stichprobengröße von über 40 verschiedenen wissenschaftlichen Arbeiten, die in wissenschaftlichen SCI-rangierten Journals seit dem Jahr 1985 veröffentlicht wurden. Neben dem SCI-Ranking wurde eine Reihe von Mindestanforderungen für die Ergebnisse der Materialzusammensetzung festgelegt, die für die weitere Analyse in Betracht gezogen wurden. Für die vorliegende Arbeit wurden folgende Probentypen von Leiterplatten berücksichtigt: Leiterplatten von PCs, Leiterplatten von Mobiltelefonen und gemischte Leiterplatten-Proben. Die Leiterplatten von anderen Vorrichtungen, das sind PCBs von Druckern, Laptops, DVD-Playern usw., wurden mangels genügender Datenverfügbarkeit nicht in die Analyse einbezogen, wodurch eine weitere statistische Analyse nicht möglich war.

\subsection{Einfluss der technologischen} Entwicklung auf Wertmetallgehalte

Vor mehr als 60 Jahren hat IBM den ersten kommerziellen Computer mit einem Magnetplattenspeicher vorge- stellt, i. E. dem ersten Festplattenlaufwerk (Ruemmler und Wilkes 1994). Im Jahr 2016 betrug die Gesamtspeicherkapazität ca. 1600 Exabytes weltweit mit einer Wachstumsrate von $40 \%$ pro Jahr (IDC 2014), und trotz der Entwicklung neuer Technologien, z.B. Solid-StateDrive, Computer-Clouding etc., sind Festplattenlaufwerke die dominierende Lösung für die Datenspeicherung geblieben.

Die Entwicklung von Festplattenlaufwerken kann seit ihrer Ersterscheinung auf dem Markt arbiträr in vier Phasen aufgeteilt werden. Diese vier Phasen der technologischen Entwicklung können basierend auf mehreren Schlüsselmerkmalen unterschieden werden, z.B. Design, Speicherkapazität und Datenspeicherdichte. Die erste Phase dauerte von der ersten kommerziellen Anwendung bis zum Jahr 1991 und zeichnet sich durch eine große Vielfalt an Designlösungen aus. Der Beginn der zweiten Phase markiert die Einführung eines magnetoresistiven Lese-/Schreibkopfes im Jahr 1991 (vgl. Ruemmler und Wilkes 1994). Dies ist auch die Zeit, in der Festplattenlaufwerke ihr erkennbares Design erhielten, das auch heute noch vorherrscht. 

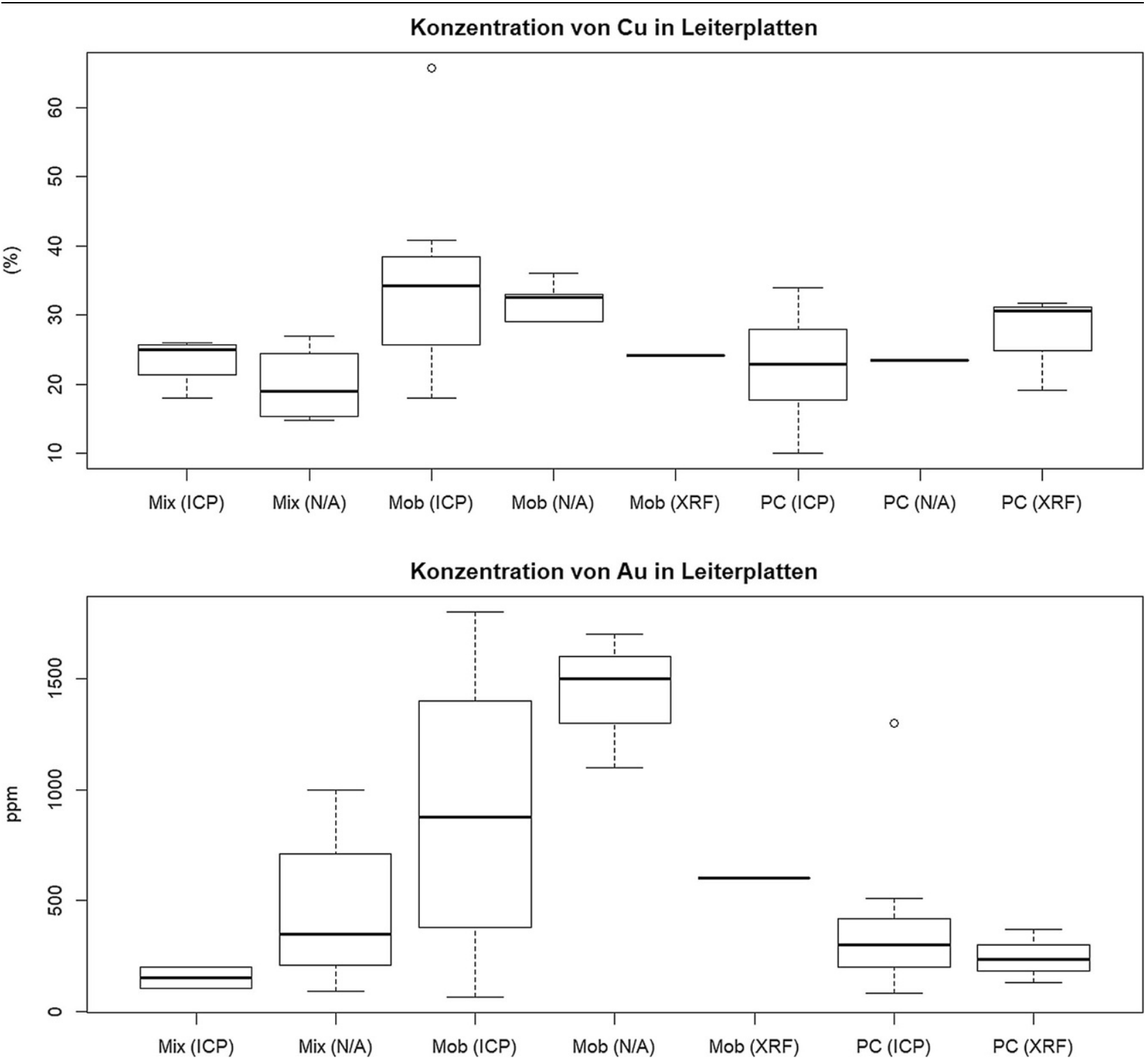

Abb. 8 Überblick von Materialzusammensetzungen von Leiterplatten (Jandric et al. 2018). Mix Gemischte Leiterplattentypen, Mob Mobiltelefon Leiterplatten, $P C$ Leiterplatten aus PCs, ICP Inductively coupled plasma, N/A unbekannt, XRF Röntgenfluoreszenz, TGA Thermogravimetrie

Die dritte und vierte Phase sind kürzer als die ersten beiden. Die dritte Phase beginnt rund um das Jahr 2000 durch das Erreichen der physikalischen Grenze der longitudinalen Aufzeichnungsart. Als Ergebnis nahm die Zunahme der Flächenaufzeichnungsdichte signifikant ab (cf. Radding 2010; Wood 2009). Als Beginn der vierten Phase in den Jahren 2005 bis 2007 wird die Entwicklung einer senkrechten Aufnahmeform angenommen. Die senkrechte Aufnahme zeichnet sich durch eine orthogonale Positionierung einer Magnetisierungsachse in Bezug auf die Plattenoberfläche aus und ermöglicht somit das Schreiben sehr scharfer Übergänge in ein Medium mit hoher Koerzitivfeldstärke (Wood 2009). Als Ergebnis dieser Innovationen hat sich die Speicherkapazität seit den 1990er-Jahren dramatisch erhöht, während das Arbeitsprinzip von Festplattenlaufwerken und deren Materialzusammensetzung relativ konstant blieben (vgl. Abb. 4).

Das Netzteil ist der Bauteil, welcher für die Stromversorgung eines PCs zuständig ist. Die primäre Aufgabe von Netzteilen besteht darin, den Wechselstrom, der von einem öffentlichen Stromnetz geliefert wird, in Gleichstrom umzuwandeln, der von den elek- tronischen Bauteilen im PC verwendet wird (Torres 2008). Der Wechselstrom aus dem öffentlichen Stromnetz hat normalerweise 110 oder $220 \mathrm{~V}$ Wechselspannung, die von dem Netzteil in kontinuierliche Spannungen von $+3,3 \mathrm{~V},+5 \mathrm{~V},+12 \mathrm{~V}$ und $-12 \mathrm{~V}$ umgewandelt wird, die von den elektronischen Bauteilen benötigt werden. Die Sekundärfunktion des Netzteils ist ein Kühlprozess für den PC (Katzer 2008; Torres 2008). Es gibt zwei Haupttypen von Netzteilen: lineares Netzteil und Schaltnetzteil, normalerweise nur als SMPS (engl. Switch Mode Power Supply) bezeichnet. Die linearen Netzteile werden 
für Vorrichtungen verwendet, die einen geringen Leistungsbedarf haben, z. B. Scanner, Drucker, schnurlose Telefone usw.

Eine Eingangswechselspannung ist umgekehrt proportional zu der Größe eines Transformators und den Kapazitäten eines Elektrolytkondensators im Netzteil. Dies bedeutet: Je höher der Strombedarf, desto größer muss der Transformator sein (Torres 2006). Da PCs einen relativ hohen Strombedarf aufweisen, sind die in PCs eingebauten Netzteile immer vom SMPS-Typ, da lineare Netzteile unpraktisch groß wären. Im Gegensatz zu Festplattenlaufwerken und optischen Laufwerken ist die Technologie hinter dem SMPSNetzteil seit dem Durchbruch der PCs konsistent, sodass die Änderungen im Design und in der Materialzusammensetzung relativ gering sind.

Da die Leiterplatten nicht im engeren Sinne funktionale Bauteile sind, sondern vielmehr eine Plattform, auf der verschiedene funktionale Bauteile montiert werden können, gibt es keine spezielle Funktion, die sie in einem elektronischen Gerät bereitstellen. Aus diesem Grund ist auch ihre Materialzusammensetzung und technologische Entwicklung gerätespezifisch.

\section{Schlussfolgerung}

Als Hauptvoraussetzung für weitere Prozessoptimierung benötigen die Vor- behandlungsunternehmen möglichst präzise Daten über die Materialzusammensetzung der EAGs und, wenn möglich, praktikable, zuverlässige und kostengünstige Analysemethoden für die Eingangscharakterisierung. Auf diese Weise wäre eine Optimierung und Effizienzsteigerung des gesamten Recyclingprozesses möglich, womit das Potenzial des Materialeinsatzstroms einfacher abgeschätzt werden kann.

Die Ergebnisse der manuellen Tiefenzerlegung und anschließenden einfachen Materialidentifikationsanalysen zeigen, dass sich diese Vorgangsweise gut für die Analyse komplexer Bauteile aus EAGs eignet. Mit dieser Methode können mehr als acht verschiedene Basismetalle oder mehr als zehn verschiedene Materialien identifiziert werden. Darüber hinaus ist es mittels dieses Verfahrens möglich, die Änderungen der Trends in der Materialzusammensetzung von komplexen Bauteilen darzustellen und genau $\mathrm{zu}$ bestimmen. Die komplexen Bauteile sollen nach dem Herstellungsjahr geordnet und als Summe ihrer Teile betrachtet werden. Dieses Analyseverfahren schafft eine zuverlässige Struktur in der variablen Materialzusammensetzung der komplexen Bauteile.

Nach der Auswertung der Ergebnisse konnte festgestellt werden, dass die Kosteneffektivitätsmaßnahmen (z. B. dünnere Magnete oder kleinere Bauteile) der Hersteller einen viel höheren
Einfluss auf die Trends der Materialzusammensetzung aufweisen, als die Folgen der Technologieentwicklung (z.B. Datenspeichereffizienz, Entwicklung von integrierten Schaltungen und Entwicklung von Lese-/Schreibköpfen).

Letztlich lässt sich aus der Anwendung der einfachen Materialidentifikationstests auch ableiten, dass eine umfassendere Methode bzw. eine Kombination von verschiedenen Tests zur Unterscheidung von gelben/roten Metallen in EAGs, d.h. Bronze, Messing und Kupfer, die Gesamteffizienz der vorgeschlagenen Materialzusammensetzung noch erhöhen würde.

Funding Open access funding provided by University of Natural Resources and Life Sciences Vienna (BOKU).

Open Access Dieser Artikel wird unter der Creative Commons Namensnennung 4.0 International Lizenz (http:// creativecommons.org/licenses/by/4. 0/deed.de) veröffentlicht, welche die Nutzung, Vervielfältigung, Bearbeitung, Verbreitung und Wiedergabe in jeglichem Medium und Format erlaubt, sofern Sie den/die ursprünglichen $\mathrm{Au}$ tor(en) und die Quelle ordnungsgemäß nennen, einen Link zur Creative Commons Lizenz beifügen und angeben, ob Änderungen vorgenommen wurden.
Baldé, C.P., Wang, F., Kuehr, R., Huisman, J. (2015): The global e-waste monitor - 2014. In. United Nations University, IAS - SCYCLE, Bonn, Germany

Chancerel, P., Meskers, C.E.M., Hagelüken, C., Rotter, V.S. (2009): Assessment of Precious Metal Flows During Preprocessing of Waste Electrical and Electronic Equipment. Journal of Industrial Ecology 13 (5):791-810. https://doi.org/10. 1111/j.1530-9290.2009.00171.x

Europäische Kommission (2017): Communication from the Commission to the European Parliament, the Council, the European Economic and Social Committee and the Committee of the Regions on the 2017 list of Critical Raw Materials for the EU. In.

Fu, J., Zhou, Q., Liu, J., Liu, W., Wang, T., Zhang, Q. Jiang, G. (2008): High levels of heavy metals in rice (Oryzasativa L.) from a typical E-waste recycling area in southeast China and its potential risk to human health. 71 (7):1269-1275. https:// doi.org/10.1016/j.chemosphere.2007.11.065

Habib, K., Parajuly, K., Wenzel, H. (2015)

Tracking the Flow of Resources in Electronic Waste - The Case of End-of-Life Computer Hard Disk Drives. Environmental Science \& Technology 49 (20):12441-12449. https://doi.org/10. 1021/acs.est.5b02264
Hagelüken, C., Corti, C.W. (2010): Recycling of gold from electronics: Cost-effective use through ,Design for Recycling،. Gold Bulletin 43 (3):209-220. https://doi.org/10.1007/ bf03214988

Huisman, J., Delgado, C., Magalini, F., Kuehr, R., Maurer, C. (2007): 2008 Review of Direc tive 2002/96 on Waste Electrical and Electronic Equipment (WEEE) - Final report. In.

Huisman, J, van der Maesen, M., Eijsbouts, R.J.J., Wang, F., Baldé, C.P., Wielenga, C.A. (2012): The Dutch WEEE Flows. United Nations University, ISP - SCYCLE, Bonn, Germany IDC (2014): Executive Summary: Data Growth Business Opportunities, and the IT Imperatives | The Digital Universe of Opportunities: Rich Data and the Increasing Value of the Internet of Things. EMC Digital Universe with Research \& Analysis by IDC

Jandric, A. (2017): Material composition of complex compoenents in WEEE. University of Natural Resources and Applied Sciences Vienna, Vienna

Jandric, A., Tran, D.C., Beigl, P., Micuda, Z., Salhofer, S. (2018): Material composition and informal treatment of Printed Circuit Boards. In precessing
Katzer, C. (2008): Debunking Power Supply Myths.

Khanna, R., Cayumil, R., Mukherjee, P.S., Sahajwalla, V. (2014): A Novel Recycling Approach for Transforming Waste Printed Circuit Boards into a Material Resource. Procedia Environmental Sciences 21:42-54. https://doi.org/10.1016/j. proenv.2014.09.006

Luidold, S, Kozlik, M., Raith, J, Janisch, A, Moser, P., Treimer, R. (2013): Kritische Rohstoffe für die Hochtechnologieanwendung in Österreich. In. Bundesministerium für Verkehr, Innovation und Technologie, Wien, Österreich

München, D.D., Veit, H.M. (2017): Neodymium as the main feature of permanent magnets from hard disk drives (HDDs). Waste Management 61:372-376. https://doi.org/10.1016/j.wasman. 2017.01.032

Radding, A. (2010): Hard disk drive technology trends - Storage Technology Magazine. http:// searchstorage.techtarget.com/magazineContent/ Hard-disk-drive-technology-trends

Ruemmler, C., Wilkes, J.W. (1994): An introduction to disk drive modeling. IEEE Computer 27(3):17-29, March 1994 It supersedes HP Labs technical reports HPL-93-68 rev 1 and HPL-OSR-93-29 27 
Salhofer, S, Tesar, M. (2011): Assessment of removal of components containing hazardous substances from small WEEE in Austria. J Hazard Mater 186 (2-3):1481-1488. https://doi.org/10. 1016/j.jhazmat.2010.12.030

Salhofer, S., Steuer, B., Ramusch, R., Beigl, P (2016): WEEE management in Europe and China - A comparison. Waste Management 57:27-35. https://doi.org/10.1016/j.wasman.2015.11.014

Schluep, M., Hagelueken, C., Kuehr, R., Magalini, F., Maurer, C., Meskers, C., Mueller, E., Wang, F. (2009): Recycling - from E-waste to Resources. In. United Nations Environment Programme \& United Nations University, Oktoberdruck AG,

Berlin, Germany
Sprecher, B., Kleijn, R., Kramer, G.J. (2014): Recycling Potential of Neodymium: The Case of Computer Hard Disk Drives. Environmental Science \& Technology 48 (16):9506-9513. https://doi.org/10.1021/es501572z

Torres, G. (2006): Anatomy of Switching Power Supplies. In. Hardware Secrets. http://www. hardwaresecrets.com/anatomy-of-switchingpower-supplies /

Torres, G. (2008): Everything You Need to Know About Power Supplies. In. Hardware Secrets,

Tran, C.D., Salhofer, S.P. (2016): Analysis of recycling structures for e-waste in Vietnam. Journal of Material Cycles and Waste Management:1-17. https://doi.org/10.1007/s10163-016-0549-1
Ueberschaar, M., Rotter, V.S. (2015): Enabling the recycling of rare earth elements through product design and trend analyses of hard disk drives. Journal of Material Cycles and Waste Management 17 (2):266-281. https://doi.org/10 1007/s10163-014-0347-6

Wood, R. (2009): Future hard disk drive systems. Journal of Magnetism and Magnetic Materials 321 (6):555-561. https://doi.org/10.1016/j. jmmm.2008.07.027

Worldometers (2017): Worldometers - real time world statistics. 